\title{
Anesthesia in liver resections: review
}

\section{Summary}

Advances in anesthetic and surgical, new technologies and deep knowledge pathophysiological techniques have allowed in recent decades transform liver resections in safe surgery with low morbidity and mortality. The reduction of intraoperative bleeding was the main factor contributing to consolidate a high survival rate, standardizing technical and encouraging a greater number of procedures worldwide. Reducing the volume of intrahepatic blood is the pillar of this intervention, because since its application began to perform liver surgery without significant bleeding. Anesthetic strategies as reducing PVC or increased variation of combined systolic (VPS), when necessary, with the use of surgical Pringle maneuver or total vascular exclusion stimulated surgeons progress in Collisions of increasingly sophisticated and complex liver, unimaginable only a few years ago.

In addition to highly complex surgeries, they have adopted common to all her techniques, based on the universal concept of fast track and ERAS (enhanced recovery after surgery) for controlling and optimizing perioperative condition of patients. The use of protective ventilation, glycemic control, peritoperatorios correct replacement fluids, and preservation of renal function and a placement cardiovacular an effective pain treatment have resulted in a clear decrease of mechanical ventilation, ICU stay and hospital stay. In this presentation techniques that can be used today for liver resection of varying complexity, with a high degree of safety they are described.
Volume 8 Issue 5 - 2017

\author{
Francisco Carlos Bonofiglio \\ Department of Anesthesia, Hospital Italiano de Buenos Aires, \\ Argentina
}

Correspondence: Francisco Carlos Bonofiglio, Department of Anesthesia, Hospital Italiano de Buenos Aires, Argentina, Email Francisco.bonofiglio@hospitalitaliano.org.ar

Received: December 29, 2015 | Published: September 20, 2017

\section{Introduction}

Bleeding was the most common and feared complication in liver resections because of its importance depended overall patient outcomes. ${ }^{1}$ Blood loss in surgery for liver occurs by different factors, however, we can mention two as the most important: it is the largest internal organ blood volume and is related and is crossed by large vessels. There are a number of publications that have shown a direct relationship between units transfused to a patient and postoperative complications. For this reason, over the last 20 years it has developed an intensive research in order to drastically reduce bleeding in this type of intervention. ${ }^{2}$ Currently, the surgical approach of the liver is a frequent intervention in the operating room, thanks to the combination of traditional surgical and anesthetic strategies, new scientific and technological knowledge. 3 Bleeding in the current liver surgery performed by an experienced team, it is observed only in a few cases and rarely of great magnitude. In correlation with this reality, blood transfusion, is also a rare indication whether the knowledge and experience gained in this field are carefully applied. ${ }^{4,5}$ This article reviews the current techniques, with more emphasis in anesthetic procedures performed for safe liver surgery.

\section{Anesthetic and surgical strategies to decrease bleeding}

Anatomy and Physiology of liver with surgical and anesthetic importance: The liver is the body's largest solid organ that is located in the right quadrant of the abdomen and is divided into four lobes: right, left, square and caudate. The right and left lobes are divided into segments, defined by the distribution of the arterial vessels and biliary tree.

In 2000, a meeting of experts, with the aim of creating a de initive nomenclature, describing the different interventions of the liver was performed. Today is the most widely accepted. ${ }^{6,7}$ Liver surgery can be performed according to the lobar and segmental distribution or without respecting this division. Therefore, resection of a liver lobe hepatectomy is called right or left. In turn, it is called
Segmentectomy resection of a single segment and when there are two, bisegmentectomy. The surgery involves removal of the right or left hepatic lobe resection plus contralateral one or two segments, it is called hepatic trisegmentectomy. ${ }^{6}$

It is described as atypical liver resection, when the cut on liver tissue, respects no segmental distribution. Liver perfusion is organized by a double movement: portal vein (PV) and hepatic artery (AH). The AH smaller caliber, delivering about $30 \%$ of the hepatic blood low with $60 \%$ of oxygen available to the body. The VP complements $40 \%$ of the total oxygen available to $70 \%$ of the low. ${ }^{9}$ The branches of the portal vein branch within the liver and blood that transports is collected by the center-lobular vein. The artery has sphincters that are part of the regulation of hepatic flow. When the portal flow decreases, it complements the hepatic artery, increasing its volume. The hepatic artery maintains a self-regulating system, which remains constant infusion, despite variations in systemic pressure. ${ }^{9}$

This allows the liver to function even under adverse circumstances, such as sustained hypotension. After crossing the hepatic sinusoids, blood from the arterial circulation and portal it is collected by three hepatic veins, which drain into the vena cava. The hepatic low is equivalent to the delivery of $1500-1800 \mathrm{ml}$. blood per minute. ${ }^{8}$ This volume explains the perceived bleeding imporant when resection of the liver is decided.

Liver endothelium and relationship with central venous pressure (PVC): Vascular endothelium has fenestrations of a variable diameter between 100 and 500 nanometers. The importance of these holes is that it allows direct contact of the blood perfusing the liver, the organ gap, preventing any defense against changes in hydrostatic pressure. ${ }^{8,9}$ Because there is a system of self-flow, there is no possibility that the liver receive dangerously excessive flow volume increases. However, this can happen when the PVC increases. In response to increased central venous pressure, arterioles contract and facilitate the passage of liquids to the gap, increase lymphatic flow and ascites formation. ${ }^{9}$ In addition, the liver surface can ooze, thereby expelling excess. 
Long before reaching this extreme situation, the liver becomes an extraordinary reservoir of blood by increasing its internal volume. ${ }^{8,9}$ A liver resection under these conditions implies the real possibility of increasing the risk of causing a very significant bleeding within minutes during surgery.

Pringle Manoeuvre (PM): Described by Pringle in 1908, consists in temporarily occluding the hepatic artery and the portal vein, limiting the flow of blood into the liver and an increased the venous pressure in the mesenteric territory. ${ }^{10} \mathrm{MP}$ reduces the venous return and blood pressure accordingly, the cardiovascular system slightly increases the systemic vascular resistance as a compensatory response, limiting the drop in the arterial pressure. ${ }^{11}$ In the $90 \mathrm{~s}$, the PM was used continuously, with potential damage that could occur due to hepatic ischemia. Currently, this maneuver is used alternately with hepatic reperfusion period, allowing its use for an extended time. ${ }^{11,12}$ During the PM, the lack of oxygen affects the liver cells, and macrophages mass into the vessel. These cells are producers of mediator substances as the tumour necrosis factor (TNF) and Interleukins. ${ }^{13-16}$ IL 6 has been described as the cytokine that best correlates to postoperative complications. The intermittent passage of the hepatic cells through ischemia and reperfusion period, shows the development of tolerance to the lack of oxygen with decreased cell damage. ${ }^{15}$

Total Vascular Exclusion (TVE): The classic vascular exclusion is to add the occlusion of the infrahepatic and the suprahepatic inferior vena cava to those already described for the MP. Thus prevents the backflow of blood. There are variations of this manoeuvre for example, the occlusion of the suprahepatic veins tributaries of the area to be resected to avoid the total interruption of the venous return. ${ }^{17}$ The TVE is useful in the resection of tumours adjacent to large vessels. This modality can also be intermitted, alternating periods of ischemia and reperfusion. ${ }^{18}$ The venous return drops almost $50 \%$ and, consequently, of the minute volume. Therefore, decrease the systemic arterial pressure which receives an immediate compensatory response through the increase in the systemic vascular resistance. ${ }^{19}$ The anaesthesiologist must support these compensatory mechanisms through the administration of fluids and specially with vasoactive drugs. ${ }^{19}$

\section{Anesthetic technique for liver resection}

Major surgeries generally are common pathophysiological situations. Regardless of the affected organ and surgical pathology. Handling colloids and crystalloids, hemodynamic stability, hypothermiacontrol, decreased surgical stress and inflammation, preventive ventilation, pain control and other vital techniques such as acid base homeostasis ground state they are universal behavior. The anesthesiologist must therefore implement sufficient and adequate monitoring to facilitate the control of these techniques and rapid recovery. ${ }^{20}$ Specific characteristics of anesthetic technique for hepatic resections:

a. Use of anesthetic drugs that do not alter the hepatic blood low.

b. Reduction of PVC to reduce bleeding.

c. Use of vasoactive drugs to avoid hypotension.

Any anesthesia drugs (intravenous and inhalation) that does not decrease the hepatic blood flow can be an excellent alternative to using this surgery. (Isoflurane, propofol, etc). The administration of fluids and PVC increased intrahepatic increases the volume of blood, causing greater blood loss during transection of the organ. PVC decrease implies intraoperative reduce bleeding. Its value should be below $5 \mathrm{~cm} \mathrm{H}_{2} \mathrm{O}$, before resection and ideally below 3 (personal experience). ${ }^{21}$ To achieve this goal: the first step is to impose the restriction of crystalloid infusion from the start of the intervention. Often this measure is not enough in itself and must be used diuretics, especially young people, who have strong compensatory mechanisms.

Overall furosemide $(0.5 \mathrm{mg} / \mathrm{kg})$ is used in a single dose, after obtaining the first record of intraoperative PVC. Its use necessarily implies the serial control blood potassium levels, which should be corrected to maintain normal levels. Use this drug has the additional effect of protecting the kidney, when vena cava clamps are used. It has also been described to decrease intrahepatic blood volume, drug with venous action as nitroglycerin, but it is more difficult to control systemic pressure. Regular use of vasoactive drugs necessary, while low PVC is maintained to maintain hemodynamic stability. Vasoactive drugs to be used at this operating time, should provide even increase compensatory mechanisms. Phenylephrine is the drug of choice because it selectively acts on vascular resistance. It is infused when PVC begins to decrease $(0.2$ to $0.3 \mu \mathrm{g} / \mathrm{kg} / \mathrm{min})$ and is increased according to the needs. It is uncommon to use higher doses of $1 \mathrm{microgram} / \mathrm{kilo} / \mathrm{minute}$ and normally do not need to add other drugs for hemodynamic maintenance of these patients (personal experience).

When a drop of effective blood volume occurs, the body responds with compensatory mechanisms to maintain blood pressure constant. In a first phase, neurohormonal activation flow elements derived from muscle, skin and splanchnic to vital organs begins. Hypovolemia currently created by the use of diuretics and fluid restriction, can give minimum hemodynamic changes. Activation of baroreceptors induced catecholamine release leading to increased peripheral resistance. Vasoconstriction offset the fall in venous return and maintains a near-normal blood pressure. When the system renin-angiotensinaldosterone system begins to work, it increases the sympathetic effect mainly mediated by angiotensin.

The vasoconstrictor effect promotes blood flow to the brain, heart and kidney. However, if hypotension renal flow is also sacrificed deepens. ${ }^{22}$ If the current volume is reduced by $50 \%$ (does not happen with the described liver, unless technically if an abrupt and massive bleeding resections) a phase characterized by bradycardia and sudden drop in blood pressure is achieved. Although they seem related, both hemodynamic phenomena respond to different stimuli. ${ }^{22,23}$ Bradycardiabe submitted in response to stimuli that are based on intracardiac mechanoreceptors, in order to promote greater diastolic phase for better ventricular filling. ${ }^{22}$

The drop in blood pressure, due to inhibition of the sympathetic mechanism worked trying to counteract hypotension. In the patient passes seamlessly into a phase of vasodilation. ${ }^{22,23}$ When the period of hepatic resection crystalloid volume status is reset, reaching basal levels of PVC. Since the beginning of the controversy over the use of colloids in critically ill patients has preferred to crystalloids for volume replacement. ${ }^{24}$ The basic intraoperative monitoring includes hepatic resections, hemodynamic monitoring, ECG, invasive blood pressure, capnography, oximetry, control of muscle relaxation, BIS (especially if intravenous anesthesia is used), control of acid base status, and control PVC coagulation if necessary. To prevent postoperative pain, some anesthesiologists prefer to avoid the use of epidural catheters, because of the possibility of liver failure and clotting disorders immediately after surgery and for a prolonged period. A very effective alternative is to indicate the use of morphine subdural. 


\section{Minimally invasive hemodynamic monitoring}

More experience and new research begin to show that the minimally invasive monitorieo could be a very effective control both to reduce the venous volume in this intervention, to replenish fluids properly. These new mini-invasive parameters that could replace PVC in the future are: stroke volume variation and change in systolic pressure. VVP and VPS respectively, ${ }^{25}$ also they have joined in this line of safety and better patient outcomes, better control for not achieving fluid overloads in time to replenish fluids. It seems clear today that hypervolemia generates pressure on the endothelial surface to damage the liver, affecting among other things the ability to impair the passage of liquids and neutrophils from one side to another extension.

The PVC does not allow to know for sure if the patient is in sometime hypervolaemic fluid replacement. But undoubtedly, the VVP and VPS can avoid this unwanted hemodynamic status. Maintain a functional and healthy endothelium, it is to avoid a state of increased inflammation in a patient under a ma jor surgical procedure. The degree of inflammation reaching the organs and tissues in the body are closely related complications intra and postoperative evolution and especially of a patient. ${ }^{26,27}$

Two measures that should be mentioned as processes that allow reducing bleeding and the inflammatory state in liver surgery are:

a. Failure to use PEEP during the resection to increase venous return pressure on the liver, facilitates bleeding and, ${ }^{28}$

b. The systematic use of ringer lactate or balanced solution, to avoid complications attributed to colloids in critically ill patients and metabolic acidosis characteristic of the physiological solution. ${ }^{29,30}$

\section{Effects of chemotherapy on the liver}

Chemotherapy drugs more changes occur on the liver parenchyma are the oxaliplatin, Avastin and irinotecan, causing steatosis, sinusoidal obstruction and fibrosis. ${ }^{31-33}$ If they are administered before surgery, the same quality in reducing bleeding, even with proper PVC reduction is not achieved. Postoperatively, the changes of the parenchyma, as a result of chemotherapy, increase the chance of liver failure. It takes at least 6 months after the last drug application for the return parenchyma has prior characteristics. ${ }^{34}$

\section{Other strategies to reduce bleeding}

Another condition that anesthesiologists should know and keep to maintain control of hemostasis, is hypothermia. The body temperature decreases during prolonged surgeries and major incisions, the wide exposure of abdominal viscera. Control of hypothermia requires active work of the anesthesiologist, by use of thermal blankets, administering warm fluids for intravenous use and warming of inspired gases, etc. ${ }^{34}$ Other techniques in the field of anesthesiology, which help not increase bleeding in liver surgery:

i. Not put heparin, even at very low doses in the washes of the arterial roads.

ii. In The pre-anesthetic evaluation, consider whether administration of iron is needed, erythropoietin or Vitamin K, according to the patient's condition.

To study all patients with von Willebr and disease and indicate desmopressin when needed. ${ }^{35}$

\section{Technological contributions in surgery}

Surgery has also provided sophisticated equipment, to allow bloodless hepatectomy. The most used are the ultrasonic scalpel, the electrocautery and argon hemostatic fibrin material that can be placed on the surface of the liver to promote clotting. ${ }^{36}$ Arterial embolization angiography, also reduces the size of tumors before surgery and blood flow to the area where the operation is performed. It is efficient to reduce intraoperative bleeding technique. ${ }^{37}$ Another widely selected to preserve healthy tissue surrounding a malignant tumor or in difficult access areas is radiofrequency method. It consists of needles special designs that are located within the metastases, which destroy by emitting intense heat. The radio also has the advantage of allowing the treatment of unresectable tumors, with a mini-invasive technique. The ultrasound helps the placement of the needle when you decide to use radio frequency. ${ }^{38}$ Laparoscopic and robotic techniques have also been adopted for liver resection, avoiding large incisions, bleeding and low tenor of postoperative pain. ${ }^{39}$

\section{Postoperative analgesia. Fast Track ERAS}

The analgesic technique to select depends on the clinical condition of the patient and fundamentally the coagulation status at the time of administering or removing catheters placed. The current discussion is held between two options, thoracic epidural analgesia and intrathecal opioids. Thoracic epidural analgesia with catheter infusion of local anesthetics, analgesics is the highest standard in abdominal and thoracic surgery, but before liver resection, the following conditions occur:

i. In pre-cirrhotic or coagulation disorders patients, place a large bore needle into the epidural space, could facilitate bruising at the anatomic space, with development of severe neurological injury.

ii. If I had no prior coagulation disorders, they are likely to be able to register after liver resection, even in patients without cirrhosis and with normal pre laboratories. This limits the removal of the catheter, which may extend to 7 days.

iii. Recently some studies began to show that moderate liver dysfunction appears to increase the chance of bruising, above the normal average. The causes of altered coagulation profile in postoperative liver surgery include: intraoperative hemodilution, prolonged residual liver parenchyma hepatic ischemia or insufficient.

iv. The withdrawal of the epidural catheter should be preceded by coagulation studies that support the safety of the procedure. In case of the use of anticoagulants after surgery, they should follow the rules described in the procedural guidelines of patients with regional anesthesia and anticoagulation.

v. Addition of local anesthetics can be administered low doses of epidural opioids to improve the quality of analgesia.

vi. In large liver resections alter lidocaine metabolism and increases blood concentration, with the possibility of reaching toxic levels.

vii. Local anesthetics are administered in low doses (0.15\%-ropivacaine $0.2 \%)$ or bupivacaine $(0.125 \%-0.150 \%)$ $5-7 \mathrm{ml} /$ hour

viii. Placement of an epidural catheter before surgery and continuing administration of local anesthetics can help reduce PVC during resection, for its sympathetic tone mildly affected.

ix. Recent studies was associated with a greater likelihood of perioperative transfusion. 
The epidural analgesia display properly in conjunction with general anesthesia, has a low rate of complications, with significant improvements in the patient's clinical condition over time. ${ }^{40-45}$ The other analgesic technique also considered among the highest standards of treatment of acute pain is intrathecal analgesia with morphine. Spinal injection of opioids has advantages and disadvantages with respect to the epidural analgesia by local anesthetics, which should be considered according to each patient. ${ }^{46}$

\section{Advantages:}

a. Provides quality analgesia for 24 hours and decreased use of intravenous analgesics within 48 hours.

b. A single injection is given with a small gauge needle, with less bruising and possible nerve damage in patients with hepatic dysfunction

c. No risk of hematoma removal of the catheter in the postoperative period.

d. It can be easily used in hospitals with low resources.

e. Does not produce sustained hypotension.

Disadvantages: (compared with epidural analgesia with local anesthetics)

a. Produces sedation and requires further reinjection extra painkillers.

b. You can make the typical side effects of opioids: immediate and delayed respiratory depression, urinary retention, pruritus, nausea and vomiting.

The average life of action of intrathecal morphine is 18 to 24 hours, and the onset of action is between 45 and 75 minutes. The spinal morphine dose ranges from 100 to 500 micrograms useful guidance but may be a weight-related, set to 2 micrograms per kilo dose. ${ }^{47-51}$ When an epidural or intrathecal technique is not possible, analgesia depend administration of intravenous morphine, either by the technique of PCA (Patient Controlled Analgesia) or regulated by the use of administrations, with bail according degree of pain present. In these cases, remember that the metabolism of morphine in liver resections may be altered. ${ }^{52,53}$

The technique of fast track begins in Cardiovascular Surgery, and initially included almost the sole purpose of early extubation (within the first hours after surgery). Its evolution now requires a multimodal treatment of the patient which includes among other objectives, an efficient treatment of postoperative pain, perioperative fluid restriction, food and early mobilization, etc. ${ }^{54}$ The programs called ERAS (enhanced recovery after surgery) push the study and development of techniques that support the comfort of patients, decrease complications and hospitalization costs, as well as facilitating the work of the doctor and the nurses. ${ }^{55}$

\section{Liver resections special features}

Surgical teams with more experience and also performed liver transplants, surgeries can practice highly complex, involving patients with unresectable tumors with conventional techniques.

\section{Ex vivo liver resection}

The ex vivo surgery involves removing the liver from the abdominal cavity with hepatectomy technique used in liver transplantation and resection of the tumor in the liver exsangue outside the body. After the resection, liver is implanted back into his abdominal position, performing the arterial, venous and biliary anastomosis appropriate. ${ }^{56}$ In liver surgery ex vivo, when the liver is removed from the body, the blood inside is replaced by preservation solution between 0 and $4^{\circ}$, allowing an intervention of several hours without problems when reimplanted. Although the morbidity and mortality of this technique is higher than conventional liver resections, it is also true that survives a percentage of patients without this surgery has no chance of treatment. Yet there are few reports on the long-term results of this surgery and the experiences described are varied and incomplete.

\section{Some conclusions are:}

a. Kidney failure is one of the most frequent complications.

b. The anesthetic technique should NOT include the reduction of PVC: it is not required by vascular clamps and because it is difficult to maintain hemodynamic stability for lack of venous return. Hypovolemia and clampeos on the inferior vena cava above the liver are responsible for IR. ${ }^{57}$

\section{Associating Liver Partition and Portal vein Ligation for Staged hepatectomy (ALPPS)}

It is a recently introduced surgical technique that allows large liver resections, but preventing the development of liver failure. This technique can include patients who a few years ago could not be operated because the remnant liver would be very small. It consists of two operations: the first operation consists essentially in occluding right portal vein, which leads to progressive atrophy of the liver lobe receiving no normal blood flow, with consequent hypertrophy of the left liver. In the second operation resection of liver usually it is done right committed to remain a left liver, which is the longest in time and with enough functionality. You can even make partial lobe resection in the latter if there are metastases in the sector. Thus liver failure is avoided. ${ }^{58}$

In the first operation should be performed with conventional anesthetic technique reducing PVC; in order to decrease the size of the liver, allowing the proper work of the surgeon in a small sector and reduce bleeding. In the second intervention is necessary to reduce the PVC, the liver is separated into two parts, one of which (right) will be finally resected, but without significant parenchymal transection. The anesthetic technique, monitoring and postoperative analgesia are conducted, according to the described criteria for liver resections in general. ${ }^{59}$

\section{Conclusion}

The liver metastasis seat from different organs and primary tumors. This has forced surgeons to a permanent search for treatments to improve patient survival and even reach their healing. In two decades, liver surgery, is no longer a procedure reserved for very few centers and very experienced surgical teams, to become recognized as a frequent intervention and a low incidence of complications. The combination of anesthetic and surgical advances have allowed this medical success. It is possible that in the coming years can attend progress, even today we cannot imagine.

\section{Acknowledgments}

None.

\section{Conflicts of interest}

Author declares that there is no conflict of interest. 


\section{References}

1. Foster J, Berman M. Solid liver tumors. Major ProblClin Surg. $1977 ; 22: 342-343$

2. Gayowski TJ, Iwatsuki S, Madariaga JR, et al. Experience in hepatic resection for metastatic colorectal cancer. Analysis of clinical and pathological risk factors. Surgery. 1994;116(4):703-710.

3. Jarnagin WR, Gonen M, Fong Y, et al. Improvement in perioperative outcome after hepatic resection. Analysis of 1803 consecutive cases over the past decade. Ann Surg. 2002;236(4):397-406.

4. Wagner JS, Adson MA, Van Heerden JA, et al. The natural history of patients with liver metastases from colorectal cáncer. A comparison with respective treatment. Ann Surg. 1984;199(5):502-508.

5. Scheele J, Stang R, Altenodorf Hofmann A. Resection for colorectal liver metastases. World J Surg. 1995;19:59-71.

6. Strasberg S. Nomeclature of hepatic anatomy and resections: a review of the Brisbane 2000 system. J Hepatobiliary Pancreat Surg. 2005;12(5):351-355.

7. Parks F, Gelman S. Normal liver function and the hepatic circulation Capít $\mathrm{u}$ lo $\mathrm{n}$ nesth esia nd Intensive Care for patients with liver disease. Park G, KanfY (Eds.), Butterworth-Heinemann: Washington, USA; 1995

8. Paulsen A. Hepatic anatomy, physiology and assessment of hepatic function. Chapter 4. In transplantation of the liver. Busuttil R, Klintmalm G (Eds.), Saunders Company: Philadelphia, USA; 1996.

9. Lautt W. Mechanism and role of intrinsic regulation of hepatic arterial blood flow: Hepatic arterial buffer response. Am J Physiology. 1985;249(5 Pt 1):G 549-556.

10. Dixon E, Vollmer C, Bathe O. Vascular occlusion to decrease blood loss during hepatic resection. Am J Surg. 2005;190(1):75-86.

11. Delva E, Camus Y, Nordlinger B, et al. Vascular occlusions for live resections. Operative management and tolerance to hepatic ischemia: 142 cases. Ann Surg. 1989;209(2):211-218.

12. Man K, Fan ST, Ng IO, et al. Prospective evaluation of the Pringle maneuver in hepatectomy for liver tumors by a randomized study. Ann Surg. 1997;226(6):704-713.

13. Badia JM, Ayton LC, Evans TJ, et al. Systemic cytokine response to hepatic resections under total vascular exclusion. Eur J Surg. 1998;164(3):185-190

14. Kretzschmar M, KrugerA, Schirrmeister W. Hepatic ischemia reperfusion syndrome after partial liver resection. Hepatic venous oxygen saturation, enzyme pattern, reduced and oxidized glutathione, procalcitonin and interleukin 6. Exp Toxic Pathol. 2003;54(5-6):423-431.

15. Bonofiglio FC, Molmenti E, de Santibañe. Ketamine does not inhibit interleukin 6 synthesis in hepatic resections requiring a temporary porto arterial occlusion (Pringle Manoeuvre): a controlled, prospective, randomized doubléblinded study. HPB. 2011;13:706-711.

16. Koga K, Ogata M, Takenaka I, et al. Ketamine suppresses tumor necrosis factor, a activity and mortality in carrageenan sensitized endotoxin shock model. Cir Shock. 1994;44(3):160-169.

17. Evans PM, Vogt DP, Mayes JT, et al. Liver resection using total vascular exclusion. Surgery. 1998;124(4):807-813.

18. Elias D, Lasser P, Debaene B, et al. Intermittent vascular exclusion of the liver (without vena cava clamping) during major hepatectomy. $\mathrm{Br} J$ Surgery. 1995;82(11):1535-1539.

19. Delva E, Barberousse JP, Nordlinger B, et al. Hemodynamic and biochemical monitoring during major liver resection with use of hepatic vascular exclusion. Surgery. 1984;95(3):309-318.
20. Kehlet $\mathrm{H}$, Mythen $\mathrm{M}$. Why is the surgical high risk patient still at risk. $\mathrm{Br}$ J Anesth. 2011;106(3):289-291.

21. Li Z, Sun YM, Wu FX, et al. Controlled low central venous pressures reduces blood loss and transfusion requirements in hepatectomy. World J Gastroenterol. 2014;20(1):303-309.

22. Barriot P, Riou B.Hemorrhagic shock with paradoxical bradycardia Intensive Care Med. 1987;13(3):203-207.

23. Spinella P, Holcomb J. Resuscitation and transfusion principles for traumatic hemorrhagic shock. Blood Rev. 2009;23(6):231-240.

24. Annane D, Siami S, Jaber S, et al. Effects of fluid resuscitation with colloids vs. crystalloids on mortality in critically ill patients presenting with hypovolemic shock; the CRISTAL randomized trial. JAMA. 2013;310(17):1809-1817.

25. Dunki-Jacobs EM, Philips P, Scoggins CR, et al. Stroke volume variation in hepatic resection: A replacement for standard central venous pressure monitoring. Amn Surg Oncol. 2014;21(2):473-478.

26. Myburgh JA, Mythen MG. Resuscitation fluids. $N$ Engl $J$ Med. 2013;369:1243-1251

27. Zarychandski R, AbouSetta A, Turgeon A, et al. Association of Hydroxiethyl starch administrations with mortality and acute kidney injury in critically ill patients requiring volume resuscitation: A systematic review and metaanalysis. JAMA. 2013;309(7):678-688.

28. Sand L, Rizell M, Houltz E, et al. Effect of patient position and PEEP on hepatic portal and central venous pressures during liver resection. ActaAnaesthesiol Scand. 2011;55(9):1106-1112.

29. Watanabe I, Mayumi T, Arishima T, et al. Hyperlactemia can predict the prognosis of liver resectiion. Shock. 2007;28(1):35-38.

30. Butterworth J, Mythen M. Should Normal saline be our usual choice in normal surgical patients.? Anest Analg. 2013;117(2):290-291.

31. Hoff PM, Wolff RA, Xiong H, et al. Phase II trial of combined irinotecan and oxaliplatin given every 3 weeks to patients with metastatic colorectal cancer. Cancer. 2006;106(10):2241-2246.

32. Fernandez F, Ritter J, Goodwin J, et al. Effect of steatohepatitis associated with irinotecan or oxaliplatin pretreatment on resectability of hepatic colorectal metastases. J Am Coll Surg. 2005;200(6):845-853.

33. Karoui M, Penna C, Amin-Hashem M, et al. Influence of preoperative chemotherapy on the risk of major hepatectomy for colorectal liver metastases. Ann Surg. 2006;243(1):1-7.

34. Kurz A. Thermal care in the perioperative period. Best Pract Res Clinanaesthesiol. 2008;22(1):39-62.

35. Ho land J, Henny C. Bloodless (liver) surgery? The anesthetist s view. Dig Surg. 2007;24:265-273.

36. Gugenheim J, Bredt L, Iannelli A. A randomized controlled trial comparing fibrin glue and plasma jet on the raw surface of the liver after hepatic resection. Hepatogastroenterology. 2011;58(107-108):922-925.

37. Yu YQ, Xu DB, Zhou XD, et al. Experience with liver resection after hepatic arterial chemoembolization for hepatocellular carcinoma. Cancer. 1993;71(1):62-65.

38. Sutherland LM, Williams JA, Padbury RT, et al. Radiofrequency ablation of liver tumors: a systematic review. Arch Surg. 2006;141(2):181-190.

39. Ocuin LM, Tsung A. Robotic liver resection for malignancy: Current status,oncologic outcomes, comparison to laparoscopy, and future applications. J Surg Oncol. 2015;112(3):295-301.

40. Page AJ, Ejaz A, Spolverato G, et al. Enhanced recovery after surgery protocols for open hepatectomy physiology, immunomodulation, and implementation. J Gastrointest Surg. 2015;19(2):387-399. 
41. Feltracco P, Brezzi M, Barbieri S, et al. Epidural anesthesia and analgesia in liver resection and living donodhepatectomy. Transplant Proc. 2008;40(4):1165-1168.

42. Rosero EB, Cheng GS, Khatri KP, et al. Evaluation of epidural analgesia for open major liver resection surgery from a US inpatient sample. Proc Bayl Univ Med Cent. 2014;27(4):305-312.

43. Ramspoth T, Roehl A, Macko S. Risk factors for coagulopathy after liver resection. J Clin Anesth. 2014;26(8):654-662.

44. Gray J, Shankar S. Epidural anaesthesia and analgesia for liver resection. Anaesthesia. 2014;69(4):933.

45. Curatolo M. Adding regional analgesia to general anaesthesia: increase of risk or improved outcome? Eur J Anaesthesiol. 2010;27(7):586-591.

46. Roy J, Massicotte L, Sassine M, et al. A comparison of intrathecal morphine/fentanyl and patient controlled analgesia with patient controlled analgesia alone for analgesia after liver resection. Anesth Analg. 2006;103(4):990-994.

47. Rathmell J, Lair T, Nauman B. The role of intrathecal drugs in the treatment of acute pain. Anesth Analg. 2005;101(5 Suppl):S30-S43.

48. Hughes M, McNally S, McKeown DW, et al. Effect of analgesic modality on outcome following open liver surgery: a systematic review of postoperative analgesia. Minerva Anestesiol. 2015;81(5):541-546.

49. Levy BF, Scott MJ, Fawcett W, et al. Randomized clinical trial of epidural, spinal or patient-controlled analgesia for patients undergoing laparoscopic colorectal surgery. Br J Surg. 2011;98(8):1068-1078.

50. Rudin A, Lundberg J, Hammarlund Udenaes $\mathrm{M}$, et al. Morphine metabolism after major liver surgery. Anesth Analg. 2007;104(6):1409-1414.
51. Sakowska M, Docherty E, Linscott D, et al. A change in practice from epidural to intrathecal morphine analgesia for hepatopancreato biliary surgery. World J Surg. 2009;33(9):1802-1808.

52. Vallano A, Aguilera C, Arnau JM, et al. Management of postoperative pain in abdominal surgery in Spain. A multicentre drug utilization study. Br J Clin Pharmacol. 1999;47(6):667-673.

53. Carroll I, Hah J, Mackey S, et al. Perioperative interventions to reduce chronic postsurgical pain. J Reconstr Micro Surg. 2013;29(4):213-222.

54. Lei Q, Wang X, Tan S, et al. Fast-track programs versustraditional care in hepatectomy: a meta-analysis of randomized controlled trials. Dig Surg. 2014;31(4-5):392-399.

55. Tan M, Law LS, Gan TJ. Optimizing pain management to facilitate Enhanced Recovery after Surgery pathways. Can $J$ Anaesth. 2015;62(2):203-218.

56. Gringeri E, Polacco M, D'Amico FE, et al. Liver autotransplantation for the treatment of unresectable hepatic metastases: an uncommon indication. A case report. Transplant Proc. 2012;44(7):1930-1933.

57. Oldhafer KJ, Lang H, Schlitt HJ, et al. Long term experience after ex situ liver surgery. Surgery. 2000;127(5):520-527.

58. Alvarez FA, Ardiles V, de Santibañes M, et al. Associating liver partition and portal vein ligation for staged hepatectomy offers high oncological feasibility with adequate patient safety: a prospective study at a single center. Ann Surg. 2015;261(4):723-732.

59. Guan-Qi Zhang, Zhi-Wei Zhang, Wan-Yee Lau, et al. Anesthesia in Associating liver partition and portal vein ligation for staged hepatectomy (ALPPS): A new strategy to increase resectability in liver surgery. International Journal of Surgery. 2014;12(5):437-441. 